\title{
Editorial
}

\section{Educación ambiental desde culturas y territorios en conflictos socioambientales en América Latina}

\author{
Danilo Seithi Kato ${ }^{1}$ \\ Universidade Federal do Triângulo Mineiro, Brasil \\ Yamile Pedraza-Jiménez \\ Universidad Pedagógica y Tecnológica de Colombia \\ Editores Invitados
}

Vivimos un momento histórico que marcará profundamente a la sociedad. En el mismo momento en que escribimos esta editorial para el volumen 12 de la revista Praxis \& Saber, tenemos la asombrosa cantidad de 2236454 seres humanos muertos por causa de la pandemia generada por el SARS-Cov-2 - covid-19-. Solo en Brasil y Colombia, donde escriben los editores invitados de esta edición, se registran 280885 muertes, casi dos mil muertes por día en ambos países. Son vidas perdidas que superan las tasas de mortalidad de las grandes guerras en la historia de la humanidad. Confinados o no, desde nuestros dispositivos electrónicos, nos actualizamos a un ritmo acelerado y quedamos incrédulos ante la pérdida de amigos, familiares y líderes locales tradicionales. Fatalmente, cuando se publique este texto, estos números ya serán considerablemente más altos.

En muchos estados hay un colapso del sistema de salud y la previsión de camas de UCI se ha vuelto cada vez más escasa. En Manaos, estado brasileño, seguimos el drama de personas que perdieron la vida por falta, no solo de camas, sino de oxígeno. La contradicción de vivir una época en la que el bosque tropical más grande del planeta carece de oxígeno para su gente resalta un tema político involucrado en la crisis de salud. El auge de proyectos neoliberales de derecha o extrema derecha a cargo de los Estados trajo consigo las tasas más altas de deforestación y pérdida de biodiversidad jamás registradas.

1.katosdan@yahoo.com.br 
Datos del Instituto Nacional de Investigaciones Amazónicas (2018), una entidad vinculada al poder ejecutivo brasileño, muestran que la deforestación ya alcanzó el $20 \%$ de la selva, y las tasas se han acentuado en Brasil desde el inicio del actual gobierno federal. Algunos expertos han llamado al fenómeno el "efecto Bolsonaro", que tiene como una grave consecuencia la reducción de la retención de carbono por el bosque a casi cero. También se pronostica que la continuación de la degradación promoverá lo que los investigadores llaman un "punto de no retorno", que señala daños irreversibles por la rápida y violenta supresión de bosques, promovida por gobiernos con políticas económicas neoliberales y, por lo tanto, de explotación. Este escenario revela la inhumanidad de los grupos en el poder, que insisten en decidir quién debe vivir y quién debe morir, en un estado de excepción que actúa como una máquina de muerte, descrita por Achille Mbembe en su obra Necropolítica (2018).

Existe, por lo tanto, una crisis de dimensiones socioambientales que inciden en la acentuación de los problemas derivados de la pandemia. Esta crisis viene anunciada desde hace algún tiempo en varias producciones en el ámbito ambiental. Entre las distintas causas del problema de la crisis, hay un indicio de la insostenibilidad del modelo económico basado en el consumo, que se consolida con el surgimiento de una agenda política depredadora y basada en el mantenimiento del sistema económico neoliberal. Esta política excluyente imprime una correlación de fuerzas que promueven un discurso antiambientalista, bajo la defensa de un supuesto desarrollo económico, que traería progreso a los países en desarrollo. Así se reitera un discurso sobre las sucesivas pérdidas ambientales como precio que hay que pagar para lograr la modernización.

La educación ambiental [EA] puede entenderse como el conjunto de prácticas que asocian la temática ambiental a los procesos educativos provenientes de los movimientos sociales ecologistas, preocupados por el patrón de consumo y sus impactos en la sociedad postindustrial (Carvalho et al., 2011). Sin embargo, es importante agregar a esta definición las preocupaciones sobre la delimitación del discurso ambiental materializado en la EA $y$ en los diferentes significados y sentidos que revelan ideologías en disputa (Kato et al., 2020). Para el contexto de América Latina, la EA es una práctica política que promueve la transformación social, crítica y la acción emancipadora (Pedraza, 2020)

Por consiguiente, al analizar el debate sobre EA en América Latina y el Caribe, Briggs et al. (2018) señalan el enfoque en los conflictos socioambientales y su relación con los procesos educativos como sello distintivo de las producciones latinoamericanas y caribeñas. El debate sobre la perspectiva de la educación para el desarrollo sostenible [EDS], defendida por la mayoría de los países del "Norte epistemológico", y la EA, como campo que involucra una dimensión política en un contexto de intensas asimetrías sociales y sociales, están en la agenda económica.

No es posible negar las diferentes líneas de pensamiento entre EDS y EA. Además de la fuerte relación con la noción desarrollista del sistema capitalista, también hay un aparente juego de significados en torno a la palabra sostenibilidad. Si bien el horizonte educativo es el desarrollo sustentable en la primera perspectiva, la discusión de la EA latinoamericana y caribeña gira hacia la investigación colaborativa orientada al desarrollo local y a promover debates desde los territorios inmersos en intensas injusticias y racismos socioambientales 
(Briggs et al., 2018). Los investigadores del llamado "Sur epistemológico" buscan cada vez más desvelar la colonialidad que mantiene una relación explotadora con la biodiversidad en territorios considerados por los grupos hegemónicos como la periferia del sistema-mundo.

Además, la propia producción académica se limita a las lenguas dominantes -inglés, alemán y francés-. Esto impone una barrera evidente a la circulación del conocimiento muy característica de la colonialidad en sus más diversas facetas (Quijano, 2007). Así, al pensar en la EA desde América Latina y el Caribe, se está hablando desde la idea de cultura, territorio y conflictos socioambientales. El territorio se entiende en este texto no como un espacio geográfico destinado a la especulación inmobiliaria, ni como recursos económicos en un proyecto privatista y neoliberal, sino como una forma de existencia y supervivencia para las personas que allí habitan. Por lo tanto, las dimensiones culturales que cobijan esta forma de ser y resistir en dichos territorios no pueden ser ignoradas en el debate que busca combatir la colonialidad.

Al pensar en la EA como un dispositivo de insurgencia contra la colonialidad, se requiere un posicionamiento fronterizo o, como lo articula Mignolo (2020), a partir de un pensamiento liminar. Lo que defendemos con este punto es que solo los temas más urgentes y agudos de los conflictos socioambientales vividos en América Latina y el Caribe pueden promover una mirada a gnosis distintas, diferentes a aquella de la modernidad occidental como única verdad. Este posicionamiento fronterizo requiere la escucha del otro y la posibilidad de construir otras lógicas y otros mundos a partir de las distintas formas de vivir en un territorio y de organizar el metabolismo social en cada entorno, al usar procesos educativos bajo el sesgo de la inter- o la transculturalidad como formas de resistencia a la dominación colonial.

La EA como campo de conocimiento ha mostrado un conjunto de temáticas y líneas de investigación con un importante crecimiento numérico en las producciones académicas en los últimos años. El Grupo de Investigación Interinstitucional sobre el Estado del Arte en Educación Ambiental, el Proyecto EArte, construye un Banco de Tesis y Disertaciones desde hace más de una década con una colección de más de 4520 trabajos de producción académica brasileña, realizados entre 1981 y 2016. El proyecto involucra a investigadores de la Universidad Estatal Paulista -Campus Rio Claro-, la Universidad Estatal de Campinas, la Universidad de São Paulo -Campus Ribeirão Preto-, la Universidad Federal del Triángulo Minero, la Universidad Federal Fluminense, la Universidad Federal de Paraná y el Instituto Federal de São Paulo - Campus Itapetininga-.

A partir de 2019, se amplió el trabajo de investigación de vanguardia en investigación de EA con la formación de la Red Colectiva de Investigadores en Educación Ambiental Superior en América Latina y el Caribe, que cuenta con la participación de investigadores de otros países de América Latina y el Caribe como Colombia, Argentina, Chile, México y Cuba. Además de sistematizar la producción académica en los países de América Latina, y de buscar tendencias y brechas en la producción científica, el grupo busca fortalecer un colectivo de investigadores para promover la educación ambiental crítica [EAC] desde la realidad latinoamericana y caribeña.

Estas acciones en el campo académico muestran la necesidad de la sistematización de la producción de conocimiento y la mirada analítica de lo que estamos produciendo desde 
la realidad vivida. Los colectivos y redes de trabajo del "Sur epistemológico" han mostrado poderosas formas de atender las demandas de nuestro pueblo, desde nuestro territorio, al mostrar la resistencia y la lucha por superar la hegemonía eurocéntrica que se apropia de la vida tras la invasión cultural (Freire, 2015). En otras palabras, el lugar de dominación es el lugar de la cultura. Es desde la cultura que el discurso establece la diferencia colonial (Bhabha, 1998).

Nuestra intención al proponer esta temática para el número es problematizar las relaciones que se proponen para el campo de la EA y articular los temas de cultura, territorios y conflictos socioambientales como una tríada que señala caminos hacia una perspectiva de EAC y decolonial. Por eso partimos de la siguiente pregunta: ¿cómo pensar la investigación en EAC desde una perspectiva del "Sur epistemológico" para superar las relaciones de poder colonial que históricamente se han establecido en el continente latinoamericano?

En este dossier reunimos producciones centradas en el tema "EA desde culturas y territorios en conflictos socioambientales en América Latina". Pretendemos promover la difusión del conocimiento consciente de la problemática socioambiental de las últimas décadas, frente al ataque a los movimientos ambientalistas y al orden democrático con el surgimiento de gobiernos de extrema derecha con proyectos conservadores y neoliberales en varios países de Suramérica.

Visualizamos una agenda de investigación que pueda situar la investigación en EA en un escenario de intensas asimetrías socioeconómicas y sus consecuencias en las primeras décadas del siglo XXI. También pretendemos abordar investigaciones que atiendan al pensamiento latinoamericano, que considera aspectos territoriales, inter-, intra-, transculturales y decoloniales a partir de las sabidurías y cosmovisiones de pueblos originarios, campesinos, quilombolas, movimientos sociales y de todos los grupos históricamente invisibilizados y silenciados en nuestra sociedad.

En este número presentamos diez manuscritos de investigadores de diferentes países y territorios. Los resultados de la investigación incluyen países como Brasil, Colombia, Argentina y México. Además, se enfoca en los aspectos teóricos de la colonialidad en el campo de la EA y en cómo las propias instituciones de formación reproducen las voces de la colonialidad. También existen estudios que parten de conflictos socioambientales propios de determinados territorios, en gran medida, y muestran los impactos socioambientales de la instalación de proyectos de represas en ríos desde la perspectiva de los pueblos tradicionales afectados por el conflicto. En estos artículos, el debate sobre el acceso al agua y el derecho a la identidad cultural y sus territorios se evidencia a partir de los impactos del proyecto de modernización impuesto en estas regiones. La investigación de los procesos educativos involucra luchas, resistencias e insurgencias de los pueblos subordinados. También se discuten la formación del profesorado y su relación con el etnoconocimiento como vía de sensibilización sobre la diversidad cultural, así como la preocupación por la ambientalización curricular y las políticas universitarias en el contexto de los nuevos problemas de la pandemia causada por el covid-19.

En resumen, existe un esfuerzo colectivo de los investigadores e investigadoras que integran este número para señalar caminos dirigidos hacia la justicia socioambiental basada en la EAC y decolonial. Se necesita escuchar las formas de vida tradicionales en diferentes 
biomas, partiendo del problema local como una forma de garantizar la soberanía de estas comunidades, así como de promover la construcción de otras posibles relaciones entre sociedad y naturaleza, como alternativa al desarrollo capitalista explotador dominante. A continuación, se describen los artículos que contribuyeron al debate propuesto.

El artículo elaborado por Silvina Andrea Corbetta y Pablo Sessano tiene como punto de partida los conflictos socioambientales y las disputas por el bien común de diferentes grupos sociales. La discusión se ubica en Argentina, país donde se desempeñan como docentes e investigadores, pero el problema repercute en toda América Latina. Se discute la importancia de los fundamentos políticos y conceptuales para el establecimiento de políticas de EA en el sistema educativo argentino, bajo un sesgo crítico a los modelos desarrollistas de la lógica del globalismo-capitalista, colonial-moderno. Así, parten de un marco teórico que revela la forma y los contenidos de los procesos de subordinación y la eliminación de otras epistemes, cuerpos y culturas. Apoyados por el proyecto crítico intercultural y las pedagogías decoloniales, defienden la no omisión de los conflictos e injusticias ambientales vividas por los sujetos en sus territorios, para, a partir de estos, consolidar una red de personas, proyectos y programas que muestren los movimientos de los ecosistemas y las sociedades. Con el escenario pandémico de covid-19, apuntan a una necesidad aun mayor de pensar en otras pedagogías, otras lógicas, que conduzcan a una transición hacia una nueva versión del mundo.

Al abordar la colonialidad del conocimiento, María Luisa Eschenhagen, investigadora colombiana de la Universidad Pontificia Bolivariana, también señala la necesidad de un diálogo de saberes en EA y el compromiso de la universidad con la noción de medio ambiente que reproducen. Con base en autores del grupo Modernidad y Colonialidad, muestra cómo el pensamiento occidental moderno se establece como hegemonía, y la importancia de otras formas de pensamiento, especialmente la cosmovisión indígena andina, como una forma de superar los binarismos que mantienen la colonialidad del conocimiento en el contexto universitario. Además de rechazar la idea de los diálogos para consenso, aporta una visión ampliada de la noción de medio ambiente y naturaleza en una perspectiva indígena y apunta a la hermenéutica diatópica como un camino posible para un diálogo intercivilizacional.

El artículo de André Carneiro Melo y Marco Antonio Leandro Barzano, de la Universidad Estatal de Feira de Santana, Bahía, Brasil, por otro lado, destaca la relación entre EA y los conflictos socioambientales del territorio quilombola. El enunciado "si el río se acaba, se acaba la comunidad", tomado de uno de los participantes de la investigación, revela la íntima relación entre el territorio y las formas de existencia en las comunidades tradicionales. La comunidad quilombola de Barreiros de Itaguaçu enfrenta el desafío de la constante restricción de acceso al río Verde, por parte de terratenientes y construcciones de represas. Con el objetivo de aumentar la visibilidad de la comunidad y su lucha por el derecho al territorio y la valoración de la ascendencia, los autores llaman la atención sobre la necesidad de un proyecto decolonial para enfrentar la intensa hegemonía del pensamiento eurocéntrico. El conflicto a partir de las narrativas de los vecinos se configura en lo que los autores denominan "la dimensión pedagógica del racismo ambiental".

Diógenes Valdanha Neto y Pedro Roberto Jacob, investigadores que trabajan en la Universidad Federal del Triángulo Minero y la Universidad de São Paulo, respectivamente, 
también se enfocan en conflictos socioambientales y en la cultura local basados en la narrativa de resistencia de pueblos amazónicos desterritorializados. Realizan una investigación de estudio de caso en la comunidad ribereña de São Carlos do Jamari, ubicada en el estado de Rondônia, basada en entrevistas a siete residentes y a un grupo focal con cinco personas. Los autores discuten a partir de la interpretación realizada por los pobladores sobre un desastre por inundación sufrido en el 2014 por la construcción de centrales hidroeléctricas en el río Madeira, para comprender los cambios propiciados por los procesos de aprendizaje involucrados en este grupo social a partir del contexto de la injusticia socioambiental experimentada en la comunidad. Los resultados señalan la importancia de la etnoconservación en relación con la EA latinoamericana y de cómo las estrategias comunitarias involucran procesos educativos solidarios y políticamente comprometidos para enfrentar los procesos de desterritorialización.

Otra investigación que parte de conflictos socioambientales locales para discutir una EA comunitaria fue escrita por Bárbara Pelacani, Anne Kassiadou Menezes, Daniel Renaud Camargo, Marcelo Stortti y Celso Sanchez del Grupo de Estudio de Educación Ambiental del Sur en el Estado, de la Universidad de Rio de Janeiro. En su artículo informan sobre el potencial de la EA que surge del conflicto por el proyecto de construcción de la represa Guapiaçu, en Cachoeiras de Macacu, Rio de Janeiro. En asociación con el Movimiento de Afectados por Represas, informan las acciones desde un enfoque cualitativo de la investigación. Hacen una descripción analítica de las condiciones de conflicto y de la resistencia de los campesinos de la región en una estrecha articulación con procesos educativos para la superación de las opresiones y subordinaciones sufridas con la instalación de la presa.

El artículo de Karina Vieira Martins, Geilsa Costa Santos Baptista y Rosiléia Oliveira de Almeida, investigadoras de universidades ubicadas en el Estado de Bahía-Brasil, presenta una propuesta para la formación inicial de profesores de biología basada en la etnoecología en comunidades tradicionales. Argumentan que, para una formación sensible a la diversidad cultural característica del contexto escolar brasileño, es necesario un enfoque basado en el diálogo intercultural entre la cultura científica y las culturas de los sujetos involucrados en los procesos de aprendizaje. Por lo tanto, el conocimiento local y los aspectos del entorno sociocultural deben vincularse al contexto escolar, no como actividades específicas, sino de forma continua a lo largo del proceso de formación

Romualdo José dos Santos y Luiz Marcelo de Carvalho, de la Universidad Estatal de São Paulo Júlio de Mesquita Filho, se dedican a construir posibles significados y sentidos sobre la relación entre los procesos educativos y los conflictos socioambientales presentes en las disertaciones y tesis defendidas en Brasil de 1981 a 2016. Realizan un análisis documental, en un tipo de trabajo de estado del arte, centrado en el análisis dialógico de los discursos que circulan en la investigación en EA en Brasil. Al elegir los términos participación y participación y ciudadanía, los autores señalan significados y sentidos en las tesis y disertaciones analizadas, que abordan los conflictos socioambientales como principio rector de las propuestas y prácticas de EA en una perspectiva crítica. Estos principios se basan en el diálogo entre saberes, para favorecer una formación que no solo se ajuste a la vida real, sino que, al tomar conciencia de las injusticias ambientales, busque la transformación de la realidad concreta. 
El artículo de Dieison Prestes da Silveira y Leonir Lorenzetti, ambos de la Universidad Federal de Paraná, Brasil, también discute el estado del arte de la EA en Brasil, pero con un enfoque en las publicaciones sobre la EAC en el Encuentro de Investigación de Educación Ambiental, de 2001 a 2019. Los autores asumen que la EAC ayuda a promover y comprender los problemas socioambientales en sus múltiples dimensiones. Analizaron las investigaciones e identificaron límites y tendencias a partir de 898 trabajos publicados en los anales del evento, más específicamente en 41 que trataban directamente la EAC. A partir de un análisis textual discursivo de los datos, observaron que la EAC apunta a la necesidad de una nueva mirada a los temas relacionados con la naturaleza y la sociedad, en detrimento de la lógica actual. La dimensión política enfocada en la superación de problemas socioambientales por parte de los sujetos involucrados aparece como fundamento crítico para la EA, en línea con el trabajo de Santos Carvalho en tesis y disertaciones.

Tiago Zanquêta de Souza, investigador de la Universidad de Uberaba, Brasil, presenta un trabajo que se centró en la extensión popular en EA. La investigación se llevó a cabo en una comunidad de trabajo del municipio de Uberaba, estado de Minas Gerais. A partir del marco de educación popular, realiza una observación participante con los estudiantes que revelan percepciones sobre la importancia de la extensión popular en su calificación profesional y personal. Señala la importancia de la relación dialógica entre docencia, investigación y extensión popular como una manera de formar educadores ambientales dispuestos a enfrentar las opresiones vividas.

En el artículo de María Teresa Bravo Mercado, de la Universidad Nacional Autónoma de México, hay un enfoque en el tema de la ambientalización curricular. Señala una condición paradójica al reflexionar sobre la construcción de indicadores de sostenibilidad por parte de las universidades con miras a la formación de futuros profesionales. Sin embargo, en lugar de convertirse en instituciones de cambios en el escenario ambiental, lo que se observa es el mantenimiento de problemas ambientales de insostenibilidad. Además de esta contradicción, la investigadora analiza algunos lineamientos para la ambientalización curricular ante la pandemia de covid-19 y realiza algunas aproximaciones sobre el escenario actual. Por lo tanto, señala los desafíos de los nuevos problemas ambientales que surgen en el contexto de la pandemia.

Esperamos que la lectura los motive a enfrentar los desafíos que se presentan en un contexto de pandemia y necropolítica, y que las producciones contenidas en este número conduzcan a una agenda de investigación comprometida con nuestra realidad latinoamericana y caribeña, y no a la reproducción de la colonialidad disfrazada de discurso de la modernidad desarrollista.

\section{Sección de investigación y pedagogía}

Juliana Cristina Faggion Bergmann y Andréa Cesco de la Universidad Federal de Santa Catarina, Brasil, comparten sus reflexiones alrededor de la importancia que tiene para el Programa Institucional de Becas de Iniciación a la Docencia de Español en la Universidad Federal de Santa Catarina la participación de sus integrantes en espacios escolares como una experiencia que aporta a la formación de profesores reflexivos, que consideran la investigación como parte central de su formación, aspecto central para comprender el papel de la escuela pública en la formación de ciudadanos críticos y autónomos. 
Finalmente, Yulieth Nayive Romero Rincón, Rosa Nidia Tuay Sigua y María Rocío Pérez Mesa, de la Universidad Pedagógica Nacional, Colombia, a partir del mapeamiento informacional bibliográfico y del análisis documental, muestran resultados del estado del arte sobre las relaciones de emociones de profesores de ciencias de educación básica primaria y su desarrollo profesional. De esta revisión, que abarcó 39 artículos con una ventana de 10 años, se identificaron las siguientes categorías: relaciones emociones y educación en ciencias en eventos académicos, producción académica de acuerdo con el país, alcance investigativo, metodología e instrumentos, enfoques, contextos de formación y niveles educativos implicados en las diferentes comunicaciones.

\section{Referencias}

Bhabha, H. (1998). O local da cultura (Trad. M. Ávila et al.). UFMG.

Briggs, L., Trautmann, N., \& Fournier, C. (2018). Environmental education in Latin American and the Caribbean: the challenges and limitations of conducting a systematic review of evaluation and research. Environmental Education Research, 24(12), 16311654. https://doi.org/10.1080/13504622.2018.1499015

Carvalho, I., Farias, C., \& Pereira, M. (2011). A missão "ecocivilizatória" e as novas moralidades ecológicas: a educação ambiental entre a norma e a antinormatividade. Ambiente \& Sociedade, 14(2), 35-49.

Freire, P (2015). Pedagogia do Oprimido. Paz e Terra.

Instituto Nacional de Pesquisas da Amazônia. (2018). Instituto Nacional de Pesquisas da Amazônia-Floresta Amazônica reduz capacidade de absorção de carbono chegando $\grave{a}$ quase zero. http://portal.inpa.gov.br/index.php/ultimas-noticias/3275-florestaamazonica-reduz-capacidade-de-absorcao-de-carbono-chegando-a-quase-zero

Kato, D., Kawasaki, C., \& Carvalho, L. (2021). O conceito de ecossistema como delimitação espaçotemporal nas pesquisas em educação ambiental: implicações para o ensino de Ciências/Biologia. ACTIO, 5(2), 1-23. https://periodicos.utfpr.edu.br/actio/article/ view/12291

Mbenbe, A. (2018). Necropolítica (3a. ed). N-1 edições.

Mignolo, W. (2020). Histórias locais - projetos globais: colonialidade, saberes subalternizados e pensamento liminar (Trad. S. Ribeiro). UFMG.

Pedraza, J. (2020). La interdisciplinariedad en un proceso de ambientalización curricular en la Universidad Pedagógica y Tecnológica de Colombia [Tesis doctoral en prensa]. Universidad Distrital Francisco José de Caldas.

Quijano, A. (2007). Coloniality and modernity/rationality. Cultural Studies, 21(2-3), 22-32. 